\title{
WW Vulpeculae: Photographic magnitudes based on plates of the Harvard Plate Collection ${ }^{\star}$
}

\author{
C. Friedemann, H.-G. Reimann and J. Gürtler \\ Universitäts-Sternwarte, Schillergäßchen 2, D-07745 Jena, Germany \\ Received April 15; accepted April 16; 1996
}

\begin{abstract}
Photographic magnitudes are presented for the irregular variable WW Vulpeculae. They were derived from Argelander brightness estimates carried out on $N=2774$ plates of the Harvard College Observatory Plate Collection. The data set is available at the CDS and covers the period 1898 to 1966. Although the accuracy of the magnitudes is not high, the data may be useful for the study of the long-term behaviour of the light variations of the variable.
\end{abstract}

Key words: stars: individual: WW Vul — stars: variables: other — circumstellar matter

\section{Introduction}

The irregular variable WW Vulpeculae $(=\mathrm{BD}+204236$ $=\mathrm{HD}$ 344361) has been classified as a member of the Orion Population of pre-main-sequence stars. Herbig \& Bell (1988) compiled a catalogue of these stars and carried out a detailed discussion of their properties.

The coincidence of WW Vul with the infrared point source IRAS $19238+2106$ supports the hypothesis that the variable is embedded in a circumstellar dust shell. The properties of the shell were derived from model calculations carried out by Friedemann et al. (1993). The same authors investigated the light variations of the variable. The most conspicuous features in the lightcurve are the aperiodically occurring Algol-like minima with amplitudes up to $\Delta m \approx 1.5 \mathrm{mag}$ and durations of typically 2 to 15 days. The normal light seems to be nearly constant during longer intervals (for further details see Friedemann et al. 1995). The occurrence of the Algol-like minima can be explained by occultations of the star by individual circumstellar dust clouds orbiting around it (Wenzel 1969). The observed infrared excess radiation strongly supports the hypothesis of a circumstellar origin of the optical variability.

Studying the long-term behaviour of the light variations may provide a base for a statistics concerning the frequency, amplitudes, and durations of the Algol-like events resulting in an estimation about the total number of dust clouds involved in this phenomenon.

Send offprint requests to: C. Friedemann

${ }^{\star}$ The data set is available in electronic form at CDS via ftp 130.79.128.5 or http://cdsweb.u-strasbg.fr/Abstract.html

\section{Data sampling}

For a comprehensive statistical investigation we wanted to collect as many photometric data as possible from the literature (for details see Friedemann et al. 1993). In doing so, we encountered the following problem. The first extensive study of the light variation of WW Vul was carried out by Jacchia (1941), using photographic plates of the Harvard College Observatory archive. He presented in his paper characteristic parts of the lightcurve but did not publish the data set. In order to include these observations in our study of the long-term behaviour of WW Vul, one of the authors (C. F.) repeated the brightness estimates on the Harvard plates examined by Jacchia and extended them to plates not available to him. Altogether, brightness estimates have been done on 2774 plates.

The brightness estimates were exclusively performed on blue plates of the sky patrol. The plates cover different fields which partially overlap. The brightness of the variable was visually estimated according to Argelander's method and converted into magnitudes using a sequence of comparison stars whose $B$ magnitudes were measured photoelectrically by Rössiger \& Wenzel (1972) with one exception.

The uncertainty of the magnitudes reported here comes from the error of the estimate amounting to about $\Delta m \approx \pm 0.15 \mathrm{mag}$ as well as local fluctuations of the sensitivity of the emulsions and should not exceed $\Delta m_{\text {tot }} \approx$ $\pm 0.3 \mathrm{mag}$ in most cases.

Figure 1 shows a general view of the lightcurve of WW Vul based on the brightness estimates on plates of the Harvard collection. The data cover the period from 1898 


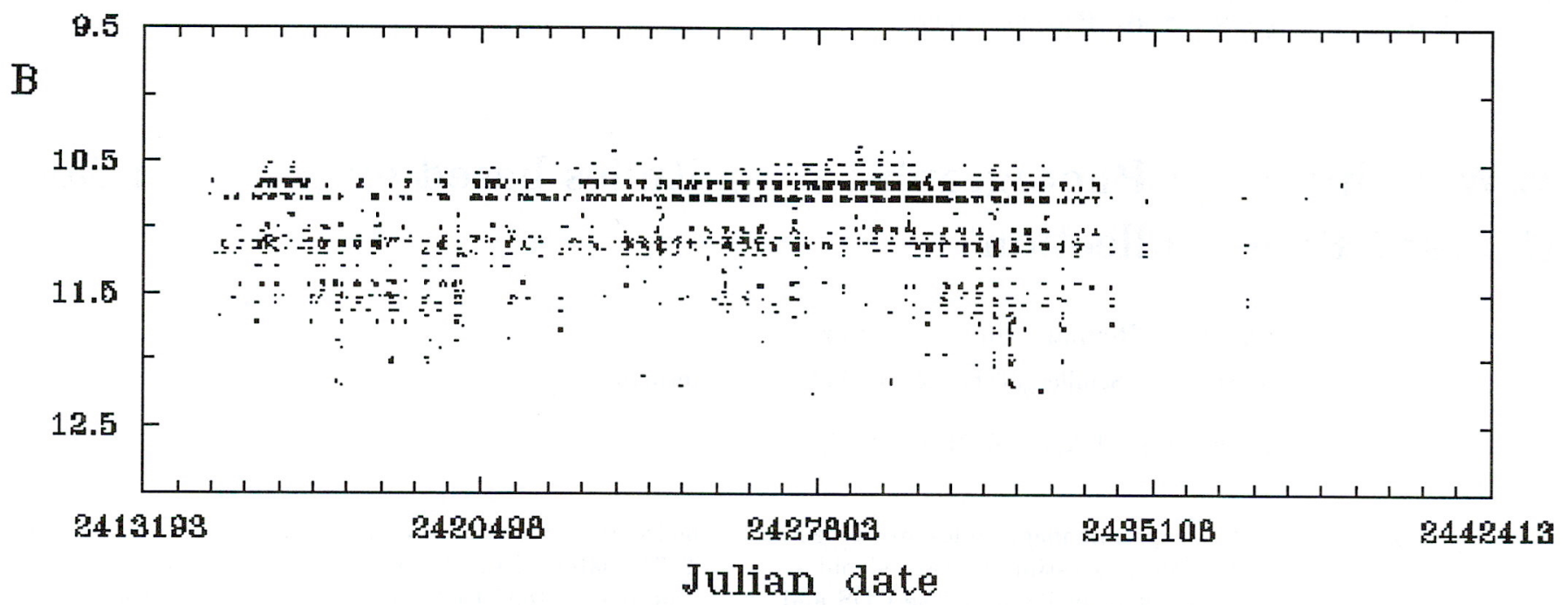

Fig. 1. Lightcurve of WW Vul based on brightness estimates on plates of the Harvard College Observatory Plate Collection covering the period 1898 to 1966

to 1966. Temporal gaps in the lightcurve are caused by seasonal effects. Horizontal stripes of missing data points in different brightness intervals have no physical meaning. A statistics of the data points reveals that the variable has virtually never been estimated to be of exactly the same brightness as the comparison star. This personal bias in the procedure of estimates produces the pattern of the empty horizontal stripes, but has no far-reaching consequences for the recognition of Algol-like minima with amplitudes larger than $\Delta m \approx \pm 0.4 \mathrm{mag}$. This minimum amplitude corresponds to about the uncertainty of the photometric data estimated above.

It is not the aim of this paper to discuss the properties of the lightcurve in detail. This has already been done elsewhere (Friedemann et al. 1993, 1995). Here we would like to call the attention to the fact that this data set is available and can be requested from the CDS Strasbourg.

We are aware that the accuracy of the magnitudes derived by visual brightness estimates is very low if they are compared with photoelectric data. Nevertheless, brightness estimates remain useful for statistical analyses of lightcurves as long as the information on the plates are not available in a digitized form.

Acknowledgements. The authors are indebted to Dr. I. Shapiro, Director of Harvard College Observatory, for the permission for using the plate collection. One of the authors (C.F.) thanks Dr. M. Hazen, Curator of the plate collection, for her kind support and hospitality during his stay. This work was partially supported by the Deutsche Forschungsgemeinschaft (Grant No. Fr 963-1).

\section{References}

Friedemann C., Reimann H.-G., Gürtler J., Tóth V., 1993, A\&A 277, 183

Friedemann C., Gürtler J., Reimann H.-G., 1995, A\&A 300, 269

Herbig G.H., Bell K.R., 1988, Lick Obs. Bull. 1111

Jacchia L., 1941, Harvard Bull. No. 915

Rössiger S., Wenzel W., 1972, Astron. Nachr. 294, 29

Wenzel W., 1969, Mitt. Veränderl. Sterne Sonneberg 5, 75 